Research Article

\title{
Population Size Estimation of Female Sex Workers in Hai Phong, Vietnam: Use of Three Source Capture- Recapture Method
}

\author{
Ly Thuy Nguyen ${ }^{1, *}, \mathbb{D}$, Shreya Patel ${ }^{2}$, Nga Thi Nguyen ${ }^{3}$, Hoa Hoang Gia ${ }^{3}$, Henry F. Raymond ${ }^{4,(\mathbb{D},}$ \\ Van Thi Hai Hoang 5 ,(1D, Abu S. Abdul-Quader, (D) \\ ${ }^{1}$ Division of Global HIV and TB Prevention, Centers for Disease Control and Prevention, Hanoi, Vietnam \\ ${ }^{2}$ The National Cancer Institute, Division of Cancer Epidemiology and Genetics, Metabolic Epidemiology Branch, National Institutes of Health, USA \\ ${ }^{3}$ Department of HIV Surveillance, Monitoring and Evaluation, Provincial HIV/AIDS Control, Hai Phong, Vietnam \\ ${ }^{4}$ Department of Biostatistics and Epidemiology, Rutgers University School of Public Health, Piscataway, NJ, USA \\ ${ }^{5}$ Department of Global Health, School of Preventive Medicine and Public Health, Hanoi Medical University, Hanoi, Vietnam \\ ${ }^{6}$ Division of Global HIV and TB Prevention, Centers for Disease Control and Prevention, Atlanta, GA, USA
}

\section{ARTICLE INFO}

\section{Article History}

Received 10 August 2020

Accepted 07 December 2020

Keywords

Population size estimate three source capture-recapture respondent-driven sampling

female sex worker

successive sampling

\begin{abstract}
Introduction: A study was conducted in three districts in Hai Phong province, Vietnam to estimate the population size of the Female Sex Workers (FSW) in June-July 2019.

Methods: The procedures included selection of three districts, compilation of a list of accessible venues where FSW congregate, distribution of first unique objects (first capture) and second unique objects (second capture) to FSW in randomly selected venues and implementation of a Mini-Respondent Driven Sampling (mRDS) Survey (third capture). Population size of the FSW was calculated based on the number of FSW in each round, number of FSW 'recaptured' during the second and the third captures. Additionally, personal network size data captured in the mRDS was used to measure the population of FSW within the three districts using Successive Sampling Population Size Estimates (SS-PSE).
\end{abstract}

Results: The total estimated FSWs in the three selected districts, using Three Source Capture-Recapture (3S-CRC) was 958, which is slightly lower than that estimated using SS-PSE - 1192. The 3S-CRC method yielded a provincial estimate of 1911 while the SS-PSE method resulted in a total of 2379 FSW for the province.

Conclusion: Two techniques produced different PSE at both the district and the province levels and resulted in estimates lower than ones produced using programmatic data. For planning HIV prevention and care service needs among all FSWs, additional studies are needed to estimate the number of sex workers who are not venue-based and use social media platforms to sell services.

(C) 2021 The Authors. Published by Atlantis Press International B.V. This is an open access article distributed under the CC BY-NC 4.0 license (http://creativecommons.org/licenses/by-nc/4.0/).

\section{INTRODUCTION}

The HIV epidemic in Vietnam is concentrated primarily among Key Populations (KP) including People Who Inject Drugs (PWID), Men who have Sex with Men (MSM), and Female Sex Workers (FSW) [1]. In the last few years, Vietnam has made significant progress in reaching 90-90-90 targets (90\% of HIV-positive persons to be diagnosed, $90 \%$ of these to be on treatment, and $90 \%$ of those on treatment achieved viral suppression) [2]. However, maintaining progress in the response to the epidemic also requires better knowledge and information about the population size and HIV burden among KP. Population Size Estimates (PSE) of KP, including those affected by HIV/AIDS, help policy makers and program administrators understand the scope of the HIV epidemic, plan appropriate interventions, and allocate enough resources. Additionally, PSE are used as

"Corresponding author.Email: ozx6@cdc.gov

Data availability statement: The datasets generated during and/or analyzed during the current study are available from the corresponding author [LTN], on reasonable request. denominators for calculating program coverage and producing national estimates and projections of the HIV epidemic. National and provincial-level authorities use these estimates to strategically plan, implement and improve disease control activities throughout Vietnam. In Vietnam, sex work is considered as "illegal" and sex workers were required to pay fines if they were caught by the police [3]. This leads to underground sex industry with sex workers living in fear of being punished and limited data are available on the number of sex workers. It is estimated that there are about 85,500 FSW aged from 15 to 49 years old [4], that represents about $0.34 \%$ of the general female 15-49 population in Vietnam in 2019 [4].

Hai Phong is the largest port city in northern Vietnam with a total population of about 2 million people [5], and it is located in the economic developmental triangle encompassing Quang NinhHai Phong-Ha Noi, the center of the HIV epidemic in the North of Vietnam. The estimated number of people living with HIV was 7000 in 2019 [4] and data from the most recent HIV sentinel surveillance [1] indicate an HIV prevalence of 5\% among FSW. 
Population size estimates of FSW, based on national program reporting system (circular 03), are available [6] (2018 estimation results presented in Table 1). Based on that, the proportion of FSW among general female 15-49 years old in Hai Phong is $0.63 \%$ [5]. However, these estimates have limitations as FSW move in and out of the province and/or change their operations. Program estimates are usually limited to those that operate out of known locations and have had contact with the services. No information is available on $\operatorname{method}(\mathrm{s})$ used to arrive at the program estimates. In the past, no rigorous attempt has been made to estimate the population size of FSW in Hai Phong. Both PWID and MSM PSE activities have recently been conducted in Hai Phong $[7,8]$. This paper describes a population size estimation activity conducted among FSW in Hai Phong. Three Source Capture-Recapture (3S-CRC) method was used to estimate the population size.

\section{MATERIALS AND METHODS}

\subsection{Three Source Capture-Recapture and Successive Sampling Population Size Estimate}

Three Source Capture-Recapture method was the primary methodology used to estimate the population size of venue-based FSW in Hai Phong [9]. 3S-CRC has been described elsewhere [10]. Briefly, 3S-CRC provides more robust estimates than the 2S-CRC and relaxes the assumption of independence between captures [10]. 3S-CRC can also be combined with a survey which serves as one of the captures. In addition, 3S-CRC produces more rigorous PSE estimates than other methods. By using Bayesian methodology, independence between capture histories can be accounted for. This

Table 1 District selection in Hải Phong for population size estimation of female sex workers

\begin{tabular}{llcccc}
\hline No & Districts & $\begin{array}{c}\text { Number } \\
\text { of FSW } \\
\text { reported } \\
\text { by national } \\
\text { program } \\
\text { system } \\
\text { 2018, } \boldsymbol{n}\end{array}$ & $\begin{array}{c}\text { Contribution } \\
\text { of each } \\
\text { district, \% }\end{array}$ & $\begin{array}{c}\text { Cumulative } \\
\text { distribution, \% }\end{array}$ & $\begin{array}{c}\text { District } \\
\text { selected }\end{array}$ \\
\hline 1 & Thuy Nguyen & 750 & 24 & & \\
2 & Ngo Quyen & 480 & 15 & 24 & Included \\
3 & Do Son & 350 & 11 & 59 & \\
4 & Hai An & 280 & 9 & 59 & Excluded \\
5 & Kien An & 280 & 9 & 68 & \\
6 & An Duong & 250 & 8 & 76 & \\
7 & Le Chan & 210 & 7 & 82 & \\
8 & An Lao & 150 & 5 & 87 & \\
9 & Hong Bang & 150 & 5 & 92 & \\
10 & Duong Kinh & 100 & 3 & 95 & \\
11 & Tien Lang & 60 & 2 & 97 & \\
12 & Cat Hai & 40 & 1 & 98 & \\
13 & Vinh Bao & 30 & 1 & 99 & \\
14 & Kien Thuy & 23 & 1 & 100 & \\
15 & Bach Long Vy & 0 & 0 & 100 & \\
& Total & 3153 & 100 & - & \\
\hline
\end{tabular}

methodology has been successfully implemented to estimate the PSE of KP in other countries such as Uganda [11] and South Sudan [12].

Implementation involved the following: the selection of the districts, training of field teams, a compilation of a list of accessible venues where FSW congregate, distribution of unique objects to FSW in randomly selected venues (first capture), distribution of a second unique object to FSW in randomly selected venues (second capture), implementation of a Mini-Respondent Driven Sampling (mRDS) survey (i.e., a community sample of FSW sampled using RDS without the extensive behavioral survey and biological testing for HIV commonly found in typical biobehavioral surveillance activities) in three selected districts (third capture).

The total population size of the FSW was calculated based on the total number of FSW in each capture, the total number of FSW 'recaptured' during the second capture, and the total number of FSWs who were recaptured during the third capture. A unique object (mirror) was distributed during the first capture while a second unique object (wallet) was distributed during the second capture. Since the third capture included a short RDS survey which included estimates of individual's personal network size, SS-PSE [13] was also used to estimate the population size of FSW within the three districts. The study was conducted in June-July 2019.

\subsection{Eligibility Criteria}

The target population included women aged 18 years or above who reported commercial sex work (selling sex to men) within the last six months and had been living in Hai Phong for the last 6 months.

\subsection{Selection of the Districts}

There are 15 administrative districts in Hai Phong, however, one (an island) is a military base not accessible to civilians. Thus, this study is limited to 14 districts. The national program report of estimated number of FSW in each district was used and ranked the 14 districts from highest to lowest. The three highest districts were selected for data collection: Thuy Nguyen, Ngo Quyen and Do Son which cover at least $50 \%$ of the total estimated FSW population in the province of Hai Phong (Table 1). Survey sites were in these selected districts to recruit FSW living and/or working in the province of Hai Phong.

After selection of the districts, field teams comprised of project staff and FSW recruited from an existing peer educator network or other non-government organizations who work with FSW in Hai Phong compiled a comprehensive list of venues where FSW congregate. Venues with a few FSW (two or three) that were in the same area were combined into a cluster before being entered into the sampling frame. The "medium" estimate was used for cluster selection. A cluster or Primary Sampling Unit (PSU) is defined as a group of 8-10 individuals who were reached in the same area. Large venues with an average of 20 or more FSW were divided to ensure all the venues had an average of 10 FSW per venue. 


\subsection{Distributions of Unique Objects}

Once the list of venues was validated and assigned a unique identification code, a random sample of venues within each district was selected for unique object distribution. Based on the lists of venues compiled, the selection of venues for unique object distribution within a selected district was made as follows:

- Step 1: number of PSU in each district = sample size of each district/average estimated number of FSW in one PSU.

- Step 2: randomly select venues from the list of venues within each district.

Once the venues were selected, the field teams visited all the selected venues and distributed the first unique object (mirror) to all eligible FSW. After obtaining informed consent, eligibility was assessed by asking FSW the following questions: their age, the last time they had sex with someone for money or goods, how long they have been living in the province and whether they have received any object distributed by outreach workers over the past week. Willing and eligible FSW received one mirror and were asked to keep it because project staff may ask about it in the near future. The object distributor log form was used to keep track of the number of unique objects distributed and contained the venue's unique identification code. At the end of the distribution, the total number of individuals that received a mirror was considered the first capture.

A week after the first capture, the second capture was conducted with the second unique object: a wallet. The venues within the selected three districts were randomly selected for the second capture. Like the first capture, the field teams assessed eligibility of the FSW with the same eligibility questions. In addition to the eligibility questions, FSW were asked the following questions: over the past 2 weeks, did you receive any object distributed by outreach workers? Can you show me? Can you describe it to me? Can you tell me how you received this object? At the end of distribution of the second object, the total number of individuals that received the first unique object (mirror) was considered re-captured. Those who received the second unique object (wallet) only were considered newly capture. The second exercise was completed in 1 week.

\subsection{Training for Field Staff}

To list the venues where FSW congregate, the field teams received orientation about the purpose of the project activity, as well as practical training on observation and confirmation of lower, upper and average estimated number of FSW in each venue. They also received training on issues related to confidentiality of the FSW and data, and safety in the field.

Prior to the distribution of the unique objects, the field teams received training on the following: assessment of eligibility prior to giving out unique objects; guidance on what to say about the unique objects; instructions on how to maintain anonymity and confidentiality; and instructions on how to maintain safety in the field. Based on the province estimate of $3153 \mathrm{FSW}$ and a sample size for the mini RDS round (200) (see below), a total of 500 objects needed to be distributed within three districts during each capture exercise (first and second capture) to estimate size of the population with a survey design effects of 2.0 and 1.96 standard deviation of the mean.

\subsection{Mini-Respondent Driven Sampling}

Two weeks after the second capture, a survey was administered in each of the three districts. mRDS is a simplified RDS-based survey that was used to gather primary data for this population size estimation study. RDS has been described elsewhere [13,14]. Briefly, RDS is a variant of chain referral sampling that uses a dual system of compensation. RDS draws on both Markov chain theory and the theory of biased networks and can reduce the biases generally associated with chain referral methods. Even though sampling begins with an arbitrarily chosen set of initial subjects (seeds), the composition of the ultimate sample is independent of those initial non-randomly selected subjects.

Three FSW were recruited as "seeds" in each district with the help of local non-governmental organizations that work with FSW. "Seeds" were selected in terms of diversity regarding the following: age, area of residence, and length of time as FSW. Each "seed" was given three referral coupons and asked to refer their peers to the survey sites in the selected districts to participate in the survey. No additional seeds were required as all seeds were able to recruit their peers.

In mRDS, participants are recruited using the RDS strategy including the coupon recruitment system linking participants; however, the key elements that differentiate mRDS from a typical RDS used in bio-behavioral surveillance surveys (BSS) include:

- Minimal field-based formative assessment focused on seed selection and distribution of unique objects.

- Elimination of the behavioral survey and the HIV testing procedures thus reducing time and cost.

- Smaller sample sizes than those for BSS.

- Administration of a brief questionnaire (approximately 10 questions) that includes screening questions with limited behavioral questions, demographics, peer network size, and population size estimation-related questions.

\subsection{Sample Size}

Sample size was calculated based on the sample needed to estimate the size of the FSW population at 95\% confidence, a half width margin of error at 0.1 , proportion of RDS respondents received the distributed unique objects of 0.4 , a design effect of 2 and a prior estimate of 3153 FSW in Hai Phong (based on the national program report). That is if we expect to be able to estimate the population size of $3153 \pm 319$. A sample size of 67 in each district achieved to reach an overall sample of 200 in three districts.

\subsection{Statistical Analyses}

Two statistical techniques were used to estimate the FSW population size in Do Son, Ngo Quyen, Thuy Nguyen: 3S-CRC and SS-PSE.

Three source capture-recapture attempts to estimate the unknown size of a closed population with different individual capture probabilities. Assumptions of the 3S-CRC methodology include: the population is closed, captures are independent, the probability of being captured is homogenous, and the matching among captures are 
accurate [9]. When heterogeneity exists among a closed population, uncertainty of the estimate will increase. To account for capture heterogeneity, 3S-CRC estimates were analyzed using a Bayesian NonParametric Latent Class Model (NPLCM) [15]. Ten latent classes where 10,000 samples from the posterior distribution were drawn with a burn-in of 10,000 iterations and a thinning interval of at least 10 iterations (20 iterations for Do Son), were used to specify the inputs of Markov Chain Monte Carlo (MCMC) sampling. Model convergence was assessed through trace plots. All analyses for 3S-CRC was performed in the R shiny application called "Multiple Source Capture Recapture" through R package LCMCR [16].

Successive sampling PSE uses a Bayesian approach, incorporating prior knowledge of the PSE and observed data collected from the RDS, to estimate the population size [17]. Reported network size, the number of recruited participants, and the date of order to survey enrollment were used to impute visibility among survey participants. SS-PSE assumes that participants with a larger network size are more likely to be recruited quickly compared to participants with a smaller network size [16]. Four network size questions (how many women you know who received money, goods, or services for sex, who live in Hai Phong and are 18 years or older and you have seen in the past 30 days) were included in the survey. Prior program estimates in 2018 were used to represent the informative prior population size. Statistical analysis for SS-PSE was performed in RDS Analyst using Gile successive sampling estimator (version 0.7). We used RDS-A to draw 1000 samples from the posterior distribution, with a burn-in of 5000 and a thinning interval at 10 iterations, and a flat distribution to specify the parameters of MCMC sampling.

\subsection{Ethical Considerations}

All procedures followed were in accordance with ethical clearance approved by local IRB (approval number: 27/HMUIRB) and US-CDC (HSR tracking number 2019-1830). Informed consent was obtained from all participants for being included in the study.

\section{RESULTS}

The number of participants approached and recruited in each district during the three captures is presented in Table 2.

The average age of participants in this study was 29.4 [Standard Deviation $(\mathrm{SD})=10.4$ ] with the youngest at 18 years old and the oldest at 58 years old. The average duration of sex work was 4.3 years $(\mathrm{SD}=5.5)$ ranging from 1.9 years within the Do Son district to 4.9 within Ngo Quyen district and 6.2 years within the Thuy Nguyen district. FSWs were sampled at various type of venues, such as massage parlors, motels, and karaoke bars.

\subsection{Do Son}

Of the 336 FSW approached during capture one, 299 accepted the mirror, 10 were ineligible, and 27 refused. Of the 335 FSWs approached during capture two, 299 accepted the wallet and 36 FSWs refused. During the mRDS (capture three) in Do Son, 67 FSW were recruited and considered captured. There were 194 captured in both capture one and two, 24 captured in both capture two and three, 24 captured in both capture one and three, and 10 FSW captured in all three captures. We estimated the number of FSW to be 504 [95\% Confident Interval (CI): 456-605] using Bayesian non-parametric latent class modeling for 3S-CRC (Table 3). The final sample size for SS-PSE analysis included 67 participants in Do Son. A prior distribution, based on a prior mean estimate of 350 , and the imputed visibility distribution, yielded a posterior median estimate of 368 (95\% CI: 87-659).

\subsection{Ngo Quyen}

Of the 106 FSW approached during capture one, all 106 were eligible and accepted the mirror. Of the 106 FSWs approached during capture two, all 106 were eligible and accepted the wallet. During the mRDS (capture three) in Ngo Quyen, 67 FSW were recruited and considered captured. There were 57 captured in both capture one and two, 37 captured in both capture two and three, 22 captured in both capture one and three, and 18 FSW captured in all three captures. The number of FSW was estimated to be 223 (95\% CI: 187-297), using Bayesian non-parametric latent class modeling for 3S-CRC (Table 3).

The final sample size for SS-PSE analysis included 67 participants in Ngo Quyen. A prior distribution, based on a prior mean estimate of 480 , and the imputed visibility distribution, yielded a posterior median estimate of 403 (95\% CI: 92-657).

\subsection{Thuy Nguyen}

Of the 97 FSW approached during capture one, 95 accepted the mirror and two were ineligible. Of the 95 FSW approached during capture two, all 95 were eligible and accepted the wallet. During the mRDS (capture three) in Thuy Nguyen, 66 FSW were recruited and

Table 3 FSW population size estimates

\begin{tabular}{lcc}
\hline \multirow{2}{*}{ Study site } & 3S-CRC & SS-PSE \\
\cline { 2 - 3 } & $\boldsymbol{n}(\mathbf{9 5 \%} \mathbf{C I})$ & $\boldsymbol{n}(\mathbf{9 5 \%} \mathbf{C I})$ \\
\hline Do Son & $504(456-605)$ & $368(87-659)$ \\
Ngo Quyen & $223(187-297)$ & $403(92-657)$ \\
Thuy Nguyen & $231(190-316)$ & $421(91-651)$ \\
\hline
\end{tabular}

Table 2 Total participants recruited in each district

\begin{tabular}{|c|c|c|c|c|c|c|c|c|c|}
\hline \multirow{2}{*}{ Study site } & \multicolumn{3}{|c|}{ Capture 1} & \multicolumn{3}{|c|}{ Capture 2} & \multicolumn{3}{|c|}{ Capture 3} \\
\hline & Approached & Eligible & Accepted & Approached & Eligible & Accepted & Approached & Eligible & Accepted \\
\hline Do Son & 336 & 326 & 299 & 335 & 335 & 299 & 68 & 67 & 67 \\
\hline Ngo Quyen & 106 & 106 & 106 & 106 & 106 & 106 & 70 & 67 & 67 \\
\hline Thuy Nguyen & 97 & 95 & 95 & 95 & 95 & 95 & 69 & 66 & 66 \\
\hline
\end{tabular}


considered captured. There were 52 captured in both capture one and two, 17 captured in both capture two and three, 16 captured in captures one and three, and seven FSW captured in all three captures. The number of FSW was estimated to be 231 (95\% CI: 190-316), using Bayesian non-parametric latent class modeling for 3S-CRC (Table 2).

The final sample size for SS-PSE analysis included 66 participants in Thuy Nguyen. A prior distribution, based on a prior mean estimate of 350, and the imputed visibility distribution, yielded a posterior median estimate of 421 (95\% CI: 91-651).

\subsection{Province Size Estimate}

The size of FSW in the province of Hai Phong was estimated using program estimates and the PSE from 3S-CRC method. The sum of program estimates of Do Son, Ngo Quyen, and Thuy Nguyen was 1580 . The sum of the remaining districts was 1573 . This ratio was applied to the estimates resulted from the 3S-CRC method, yielding a total province estimate of 1911 (Table 4). When applying this ratio to the estimates from SS-PSE method, a total of 2379 FSW were estimated for the province (Table 4).

\section{DISCUSSION}

This is the first study conducted in Vietnam from June to July, 2019 using the 3S-CRC method to estimate the population size of FSW. Also, mRDS data provided the opportunity to estimate the population size using SS-PSE technique. Total estimates for the three districts (958 FSWs) as well as for the province (1911 FSWs) - based on 3C-CRC - are lower than the program estimate, and the estimate based on SS-PSE method (1192 FSWs for three districts and 2379 FSWs for the province).

There are several reasons for the differences between the estimates based on 3S-CRC and programs and SS-PSE. Possible violations of the assumptions of the 3S-CRC activity may have produced lower size estimates than the program estimates. For example, even though there was a 1-week interval between capture rounds, it took about 3 weeks to complete the mRDS, during which some FSW may have migrated out of the survey location resulting in an underestimation of the population size. Although it is difficult to assess if independence between capture rounds was reached, the NPLCM relaxes this assumption and has the flexibility to accommodate various forms of heterogeneity among capture probabilities.

Several factors that can affect the precision and accuracy of the PSE when using SS-PSE method. SS-PSE methodology assumes

Table 4 FSW population size estimates: program, 3S-CRC and SS-PSE

\begin{tabular}{|c|c|c|c|}
\hline & \multirow{2}{*}{$\begin{array}{l}\text { Program } \\
\text { estimate }\end{array}$} & 3S-CRC & SS-PSE \\
\hline & & $n(95 \% \mathrm{CI})$ & $n(95 \% \mathrm{CI})$ \\
\hline $\begin{array}{l}\text { Do Son + Ngo Quyen + } \\
\text { Thuy Nguyen }\end{array}$ & 1580 & $958(885-1182)$ & $1192(659-2172)$ \\
\hline Other 11 districts & 1573 & $953(880-1177)$ & $1187(656-2162)$ \\
\hline $\begin{array}{l}\text { Total provincial } \\
\text { estimate }\end{array}$ & 3153 & $1911(1765-2359)$ & $2379(1315-4334)$ \\
\hline
\end{tabular}

participants with a larger reported network size have a higher probability to be recruited into the survey. Additionally, participants may not have understood the question entirely, when reporting their personal network size. For example, participants may have overestimated their personal network size by rounding.

Since this was not a traditional RDS and our sample size was very small relative to the population, leading to a lack of precision. There may have not been enough sample size in the mRDS data to inform the PSE calculated with SS-PSE. Our prior knowledge of the PSE came from program estimates which may have been biased as they rely on the visibility of FSW.

A sensitivity analysis was conducted, using flat and beta distributions to determine how the PSE was affected when modifying the prior program estimate $\pm 50 \%$. Because of the low sample size, the posterior median PSE was highly influenced by the prior knowledge of the PSE, and for that reason, a flat distribution was used.

In practice, it is unknown whether or not individuals were truly recaptured, the largest limitation of the 3S-CRC activity because unlike the other assumptions. Additionally, these estimates may be influenced by training of object distributors. Inaccurate reporting of the number of objects distributed and received by FSW may have also influenced the final PSE.

Female sex workers recruited in this study reported to meet their clients in different types of venues, including hostel, massage, karaoke bars, cafes and hotels. Majority of participants from Do Son and Thuy Nguyen were found at hostels whereas majority of FSW in Ngo Quyen district met their clients at massage parlors. The low proportion of FSW found in street venues might reflect the change in the nature of sex work, where more sex workers are based in non-street venues, making it harder to identify them and include them in PSE activities such as this one. Additionally, in Vietnam $72 \%$ population own smartphones and $45 \%$ of the population using mobile internet [18], making it increasingly likely that FSW are using these technologies to contact clients and vice versa.

\section{LIMITATIONS}

Although efforts were made to recruit FSW from different types of venues, however, only FSWs found in $60 \%$ of venues in selected districts during mapping were recruited. In the urban province such as Hai Phong, there are venues the field team were unable to approach such as certain entertainment venues or high-end restaurants. The PSE did not include those younger than 18 years and those who were very mobile. As such, those participating in this study represent a subgroup of FSW population in Hai Phong, who were accessible to the study team.

\section{CONCLUSION}

Although these estimates are lower than program estimates, the program estimates are valuable for program planning. These estimates are based on empirical data and should be considered as the absolute minimum. The methods used in the study have been well-established and used in estimating population size of KP in a variety of settings. In the absence of a gold standard PSE method, 
these estimates prove to be crucial to plan appropriate interventions and allocate enough resources. Finally, the study provided much needed experience and builds local capacity in implementing 3S-CRC PSE method that will help improve future estimation exercises.

\section{CONFLICTS OF INTEREST}

The authors declare they have no conflicts of interest.

\section{AUTHORS' CONTRIBUTION}

LTN, AAQ and HFR conceptualized the study. NTN, HHG and VTHH were responsible for filed implementation and data collection. LTN and SP analyzed data. LTN, SP and HFR provided technical support, review findings and contributed to the draft of the manuscript. All authors reviewed, made comments and contributed to the draft of the manuscript. LTN and AAQ edited and finalized the draft.

\section{FUNDING}

This study has been supported by the President's Emergency Plan for AIDS Relief (PEPFAR) through the U.S. Centers for Disease Control and Prevention (CDC) under the terms of 5U2GGH001628-02.

The findings and conclusions in this report are those of the authors and do not necessarily represent the official position of the funding agencies.

\section{REFERENCES}

[1] Vietnam Administration for HIV/AIDS Control, Ministry of Health. National HIV sentinel surveillance. Circular 09. 2018. Available from: http://vaac.gov.vn/vanban_detail/Detail/ Thong-tu-09-2012-BYT-TT-cua-Bo-Y-te-Huong-dan-giamsat-dich-te-hoc-HIV-AIDS-va-giam-sat-cac-nhiem-trung-laytruyen-qua-duong-tinh-duc.

[2] Vietnam Administration of HIV/AIDS Control (VAAC). The PEPFAR Vietnam Country Operational Plan (COP). 2020. Available from: https://www.state.gov/wp-content/uploads/2020/ 07/COP-2020-Vietnam-SDS-Final-.pdf.

[3] Huber J, France NF, Nguyen VA, Nguyen HH, Oanh KTH, Bryne E. Exploring beliefs and experiences underlying self-stigma among sex workers in Hanoi, Vietnam. Cult Health Sexual 2019;21;1425-38.

[4] Vietnam Administration for HIV/AIDS Control, Ministry of Health. The Asian Epidemic Model. 2019 [Unpublished data].
[5] General Statistics Office of Vietnam (GSO). General Statistics Office in Hai Phong. 2019 report. Available from: https:// thongkehaiphong.gov.vn/nien-giam-thong-ke/.

[6] Vietnam Administration for HIV/AIDS Control, Ministry of Health. National Program Data. Circular 03. 2018. Available from: http://vaac.gov.vn/vanban_detail/Detail/Thong-tu-03-2015TT-BYT-ve-viec-Quy-dinh-che-do-bao-cao-cong-tac-phongchong-HIV-AIDS.

[7] Des Jarlais D, Khue PM, Feelemyer J, Arasteh K, Huong DT, Oanh KTH, et al. Using dual capture/recapture studies to estimate the population size of persons who inject drugs (PWID) in the city of Hai Phong, Vietnam. Drug Alcohol Depend $2018 ; 185 ; 106-11$.

[8] Son VH, Safarnejad A, Nga NT, Linh VM, Tu LTC, Manh PD, et al. Estimation of the population size of men who have sex with men in Vietnam: social app multiplier method. JMIR Public Health Surveill 2019;5;e12451.

[9] Vuylsteke B, Vandenhoudt H, Langat L, Semde G, Menten J, Odongo F, et al. Capture-recapture for estimating the size of the female sex worker population in three cities in Côte d'Ivoire and in Kisumu, western Kenya. Trop Med Int Health 2010;15;1537-43.

[10] Thandrayen J, Wang Y. Capture-recapture analysis with a latent class model allowing for local dependence and observed heterogeneity. Biom J 2010;52;552-61.

[11] Doshi RH, Apodaca K, Ogwal M, Bain R, Amene E, Kiyingi H, et al. Estimating the size of key populations in Kampala, Uganda: 3-source capture-recapture study. JMIR Public Health Surveill 2019;5;e12118.

[12] Okiria AG, Bolo A, Achut V, Arkangelo GC, Michael ATI, Katoro JS, et al. Novel approaches for estimating female sex worker population size in conflict-affected South Sudan. JMIR Public Health Surveill 2019;5; 11576.

[13] WHO, CDC, UNAIDS, FHI 360. Biobehavioral survey guidelines for populations at risk for HIV. Geneva: World Health Organization; 2017.

[14] Heckathorn DD. Respondent-driven sampling II: deriving valid population estimates from chain-referral samples of hidden populations. Soc Probl 2002;49;11-34.

[15] Manrique-Vallier D. Bayesian population size estimation using Dirichlet process mixtures. Biometrics 2016;72;1246-54.

[16] Handcock MS, Gile KJ, Mar CM. Estimating hidden population size using Respondent-Driven Sampling data. Electron J Statist 2014;8;1491-521.

[17] McLaughlin KR, Johnston LG, Gamble LJ, Grigoryan T, Papoyan A, Grigoryan S. Population size estimations among hidden populations using respondent-driven sampling surveys: case studies from Armenia. JMIR Public Health Surveill 2019;5;e12034.

[18] Appota. Vietnam mobile app market report - First half of 2018. Available from: https://appota.com/uploads/report/Vietnam_mobile_ app_market_Report_2018_EN.pdf. 